\title{
Clinical and pathological features of patients with nemaline myopathy
}

\author{
XI YIN, CHUAN QIANG PU, QIAN WANG, JIE XIAO LIU and YAN LING MAO \\ Department of Neurology, Chinese People's Liberation Army General Hospital, Beijing 100853, P.R. China
}

Received October 18, 2013; Accepted March 18, 2014

DOI: $10.3892 / \mathrm{mmr} .2014 .2184$

\begin{abstract}
Nemaline myopathy (NM) is a rare congenital myopathy of great heterogeneity, characterized by the presence of rods in the cytoplasm of muscle fibers. This study aimed to summarize and analyze retrospectively the clinicopathological features of 28 patients with NM. Among the 28 patients, 15 were classified as of the typical congenital type, manifested as lower- or four-limb weakness as the first symptom and slowly progressive course. Six patients were classified as of childhood onset type, with lower-limb weakness and progressive course. Seven patients were classified as of the adult onset type, with rapidly progressive course and obvious muscle atrophy. Patient's 1, 16 and 23 had rapid clinical progression. On follow up, the three patients showed respiratory failure. Limb weakness in all patients was proximal-dominant. Hypotonia was observed in most patients. High arched feet were also observed as dysmorfic features. In all patients, the creatine kinase (CK) level was normal or mildly elevated, and electromyography revealed myogenic changes. Nemaline bodies were observed under a light microscope in more than half of the patients' muscle fibers, and especially in type I fibers. All patients showed fiber type I predominance and atrophy. Modified Gömöri trichrome staining showed characteristic purple-colored rods. Muscle electron microscopy revealed the presence of high electron-dense nemaline bodies around the nucleus, and of a disorganized myofibrillar apparatus, with broken myofilaments and irregular myofibrils and $\mathrm{Z}$ lines. The 28 patients with NM shared a number of clinical features, such as proximal limb weakness, reduced deep tendon reflex and dysmorfic features. Differences were also observed between the three types of patients, with regards to course progression, disease severity and respiratory failure. In conclusion, patients with NM showed great clinical heterogeneity. The diagnosis of NM was mainly based on the muscle biopsy.
\end{abstract}

Correspondence to: Mr. Chuan Qiang Pu, Department of Neurology, Chinese People's Liberation Army General Hospital, 28 FuXing Road, Beijing 100853, P.R. China

E-mail: pucq30128@sina.cn

Key words: nemaline myopathy, clinical manifestation, muscle pathology

\section{Introduction}

Nemaline myopathy (NM) is a rare congenital muscle disease with an incidence of 0.02 per 1,000 live births (1), which is characterized by the presence of rod-like structures in skeletal muscle fibers. In 1963, Conen et al (2) and Shy et al (3) were the first to describe NM. The common clinical manifestation of NM is its onset, characterized by hypotonia or general weakness predominantly affecting facial, axial and proximal limb muscles. An important clinical feature of $\mathrm{NM}$ is that weakness is more predominant in the respiratory muscles compared to other muscle groups, and this is the main cause of mortality of NM patients. According to the degree of muscle weakness, its severity and the age at onset, six types have been clinically defined by the European Neuromuscular Center (ENMC) International Consortium on NM (4): severe congenital, intermediate congenital, typical congenital, mild childhood, adult onset and other unusual types. Cases are often sporadic, but other cases with family history exhibit either autosomal recessive or dominant patterns of inheritance; mutations associated with the disease have been discovered in seven genes to date (5): TPM3, NEB, ACTA1, TPM2, TNNT1, CFL2 and KBTBD13. Although NM is a disease of high clinical and genetic heterogeneity, its diagnosis is based on standard criteria, namely, the presence of characteristic purple subsarcolemmal or cytoplasmic rods, visualized with the modified Gömöri trichrome dye and observed under a light microscope, or the presence of high electron-dense nemaline bodies of Z-line origin, observed by electron microscopy.

Since the first NM case reported by Cao et al (6) from the Beijing Friendship Hospital (Beijing, China), a total of 16 cases have been reported in China (7-16), but no study has systematically focused on the clinical and pathological features of NM. In our study, we present clinical and pathological data from a total of $28 \mathrm{NM}$ patients from China, which we used to investigate the clinical diversity and pathological features of NM.

\section{Patients and methods}

Patients and ethics. A total of 28 patients were included in this study. These patients were divided in two groups. Clinical and pathological data from a total of $28 \mathrm{NM}$ patients from China is presented and the clinical diversity 
and pathological features of Chinese patients with NM were investigated. Their enrollment was based on the references of these studies, available from the China Academic Journal Electronic Publishing House (http://www.cnki.net) and from the Medline database. This group comprised a total of 16 patients from 11 studies. The second group comprised 12 patients, admitted in the Department of Neurology at the Chinese People's Liberation Army General Hospital from 1986 until 2011. The 12 patients underwent muscle biopsy and were diagnosed on the presence of rods in the muscle fibers. Consent to analyze the muscle biopsies was obtained in the out-patient clinic or during hospital admission for diagnostic investigation. This study was approved by the hospital's Institutional Review Board.

Clinical information and laboratory examination. Study protocol data collected from each patient included: i) detailed personal history (age at onset, initial symptom or sign, motor milestone delay, clinical course and family history); ii) clinical features (distribution of muscle weakness and atrophy, progression pattern, cardiac or respiratory issues, dysphagia and mental retardation); iii) dysmorphic features (pseudohypertrophy, high arched palate and foot, elongated face and spinal deformity); iv) laboratory data [level of serum creatine kinase (CK), results from electromyography and nerve conduction velocity]; and v) muscle power (from manual muscle testing).

Electromyography. Electromyography and nerve conduction velocity tests were performed at the same time with the electromyography equipment (keypoint 2000; Dantec Dynamics, Skovlunde, Denmark). After informed consent was signed by the patients, a needle containing two fine-wire electrodes was inserted through the skin into the muscle tissue. A trained neurologist observed and recorded the electrical activity while inserting the electrode. The bilateral deltoid muscle, biceps brachii muscle, quadriceps femoris muscle and anterior tibial muscle were assessed. Spontaneous signals from a muscle at rest were measured. Then, the patients were asked to contract slightly. The shape, size and frequency of the motor-unit action potentials were recorded. In addition, the recruitment was recorded when the patients were asked to contract vigorously. In the nerve conduction velocity test, small electrical shocks were delivered to a nerve at set locations. Electrical leads attached elsewhere on the body picked up the electrical signal, and the motor nerve velocity and sensory nerve velocity were recorded.

Muscle biopsy. Open muscle biopsy was performed on the biceps brachii or the quadriceps femoris under local anesthesia. Following removal, muscle samples were immediately frozen in isopentane cooled with liquid nitrogen and were stored at $-80^{\circ} \mathrm{C}$. Transversal serial $5-\mu \mathrm{m}$ frozen muscle sections were stained with hematoxylin and eosin, modified Gömöri trichrome stain, nicotinamide dehydrogenase tetrazolium reductase (NADH-TR) and adenosine triphosphatase (ATPase) after incubation at $\mathrm{pH} 4.3,4.5$ and 10.6. Morphometric evaluation of the muscle specimens was performed under a light microscope (BX51; Olympus, Tokyo, Japan). A $0.5 \times 0.5 \times 1.0 \mathrm{~cm}$-size muscle sample was stored in glutaraldehyde and observed under an electron microscope (JEM1230; JEOL, Ltd., Tokyo, Japan). All reagents were purchased from Sinopharm Chemical Reagent Co., Ltd. (Beijing, China).

\section{Results}

Patient information. From April 1986 to December 2011, 4,127 muscle biopsies were performed on suspected myopathy cases in our hospital. The proportion of NM cases in this group was $0.29 \%(12 / 4,127)$. In the 28 patients evaluated in this study, there were 18 male $(\mathrm{M})$ and 10 female $(\mathrm{F})$, with a ratio $\mathrm{M}: \mathrm{F}=1.8: 1$. The patients' age ranged from 4 to 74 years, with a mean age $22.5 \pm 16.7$ years. The disease course ranged from 1 month to 35 years, with a mean disease course of $107.9 \pm 107.2$ months. A total of 26 patients with no family history were considered as sporadic cases, with 14 of these patients recruited from other studies and 12 from the Neurology Department in our hospital. The remaining 2 patients belong to a family with positive family history and were considered as family cases.

Clinical information. Details on each patient are shown in Table I. Among the 28 patients, 15 presented mild hypotonia and chronic limb weakness during the neonatal period, with delayed motor milestone in a few cases. Symptoms such as lower limb weakness, dyspnea and myalgia developed rapidly in 7 cases during adulthood. Three of these patients displayed rapid clinical progression and acute respiratory insufficiency, while their prognosis was poor. Lower limb weakness at childhood or adolescence was the most common initial symptom observed in the remaining 6 patients. Each of these patients leaded an independent life with mild muscle weakness. According to the age at onset, severity of muscle weakness, development of clinical progression and respiratory involvement, we classified the three groups of patients as typical congenital, adult onset and childhood onset on the basis of NM classification criteria defined by the ENMC International Consortium.

Most patients walked slowly or easily lost balance due to the lower limb muscle weakness, especially in the proximal parts. However, two patients (nos. 7 and 24) showed a different muscle weakness distribution, with higher severity of weakness observed in the distal rather than the proximal parts. Muscle weakness would then develop in the upper limb muscle, neck flexor and facial muscle groups, but cardiomyopathy and ophthalmoplegia were not detected in our study. Respiratory muscle involvement, causing respiratory insufficiency and associated with poor prognosis, was only found in adult onset type patients $(42.9 \%, 3 / 7)$.

Similar to the muscle weakness distribution, the muscle atrophy mostly occurred in the four limbs and markedly affected the proximal parts. Three out of the 7 adult onset type patients showed trunk muscle atrophy, and 9 out of the 15 typical congenital type patients showed no muscle atrophy. In 15 typical congenital NM cases, 7 showed high arched palates, 2 showed elongated faces and no dysmorphic features in other cases were identified. All patients exhibited reduced to absent tendon reflexes. Needle electromyography revealed normal nerve conduction and a combination of small, brief, 


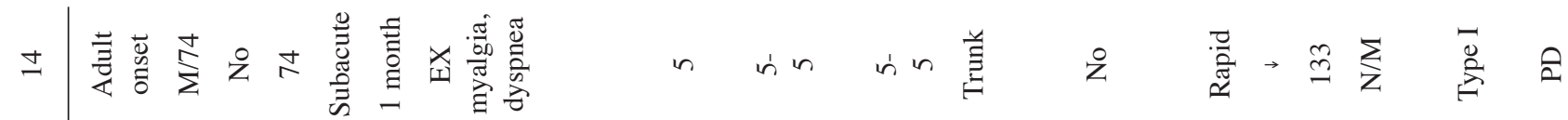

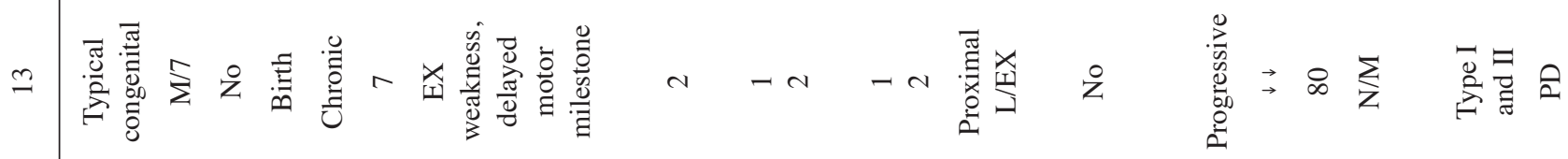

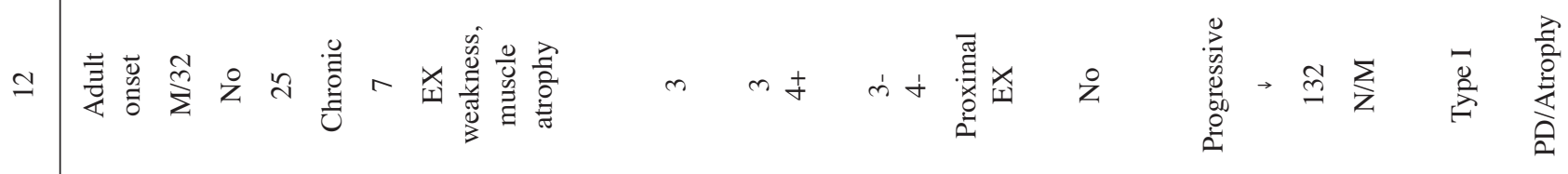

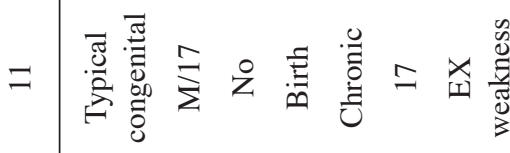

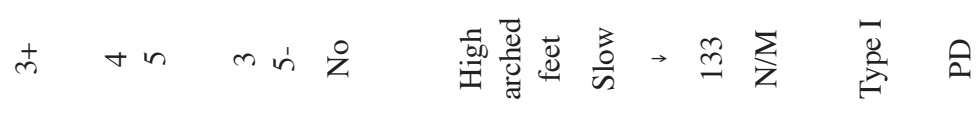

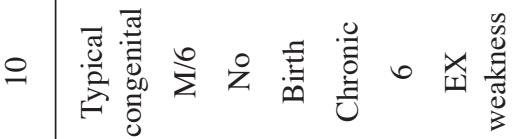

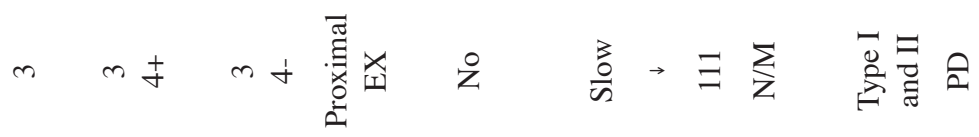

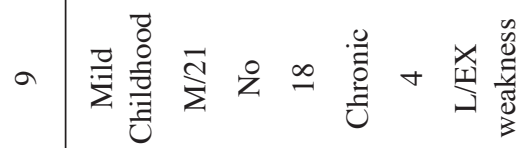

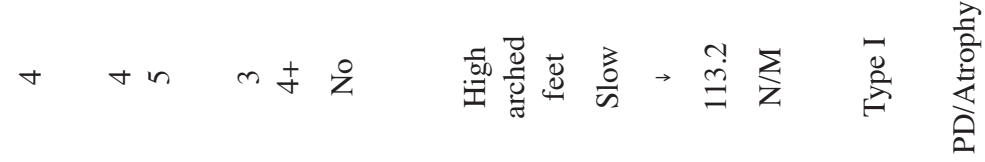

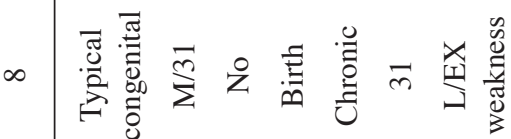

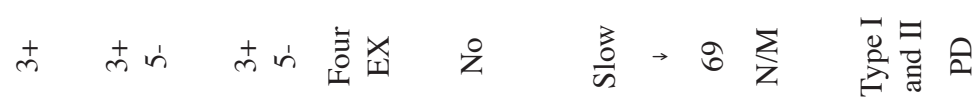

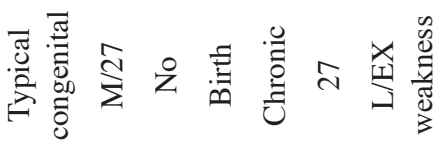

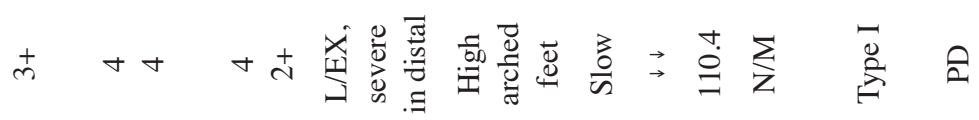

窟

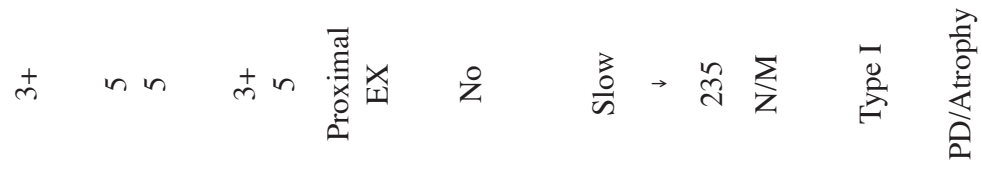

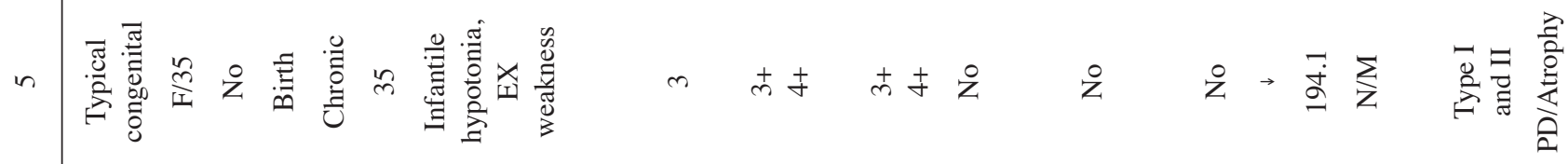

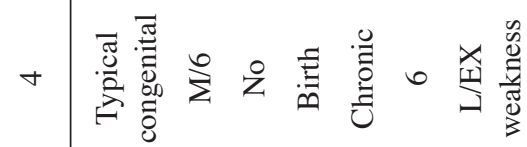

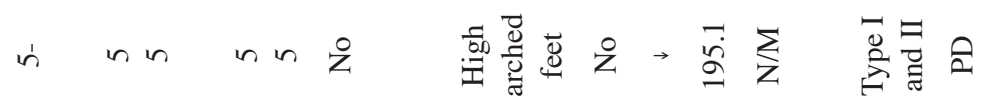

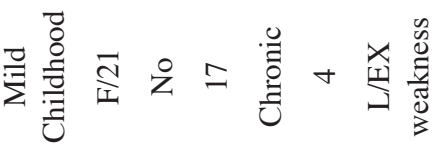

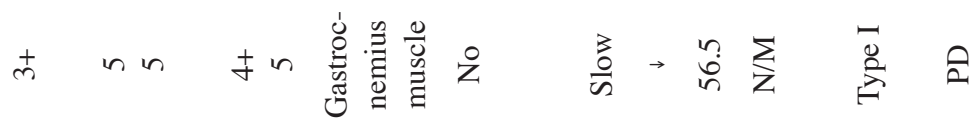

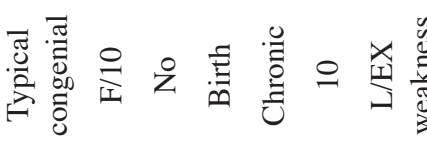

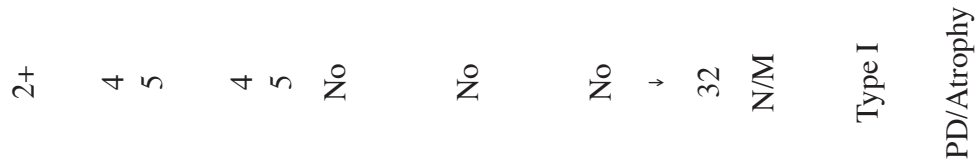

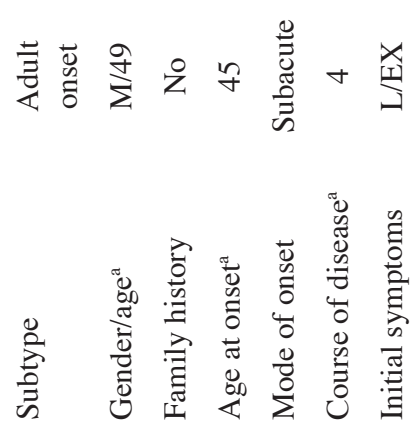

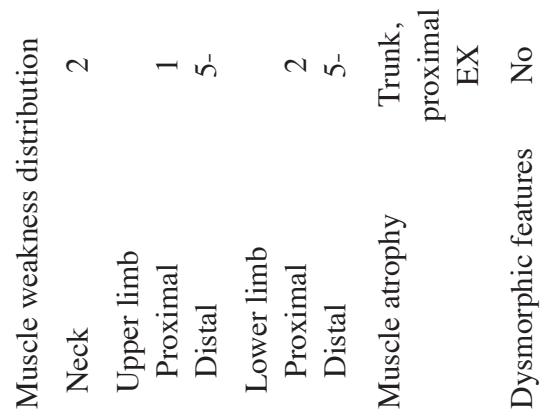

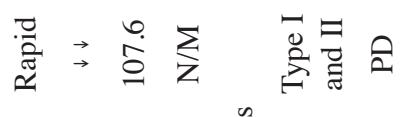

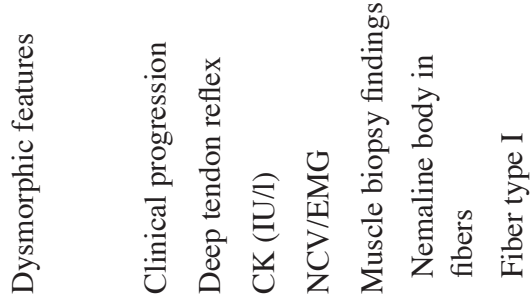




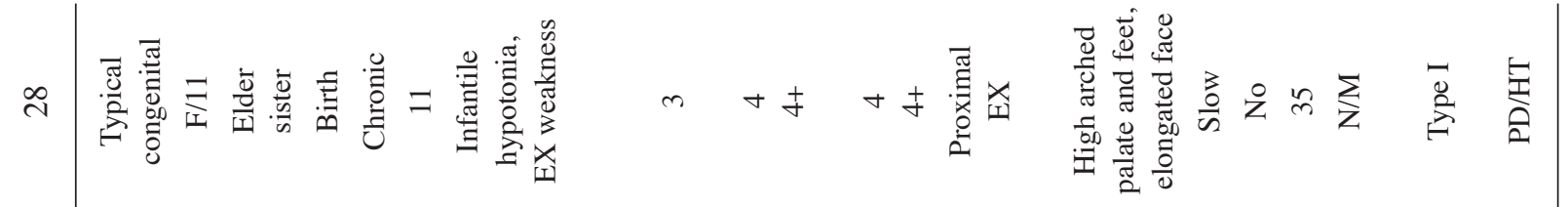

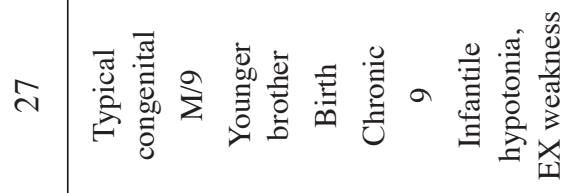
苾离蒫

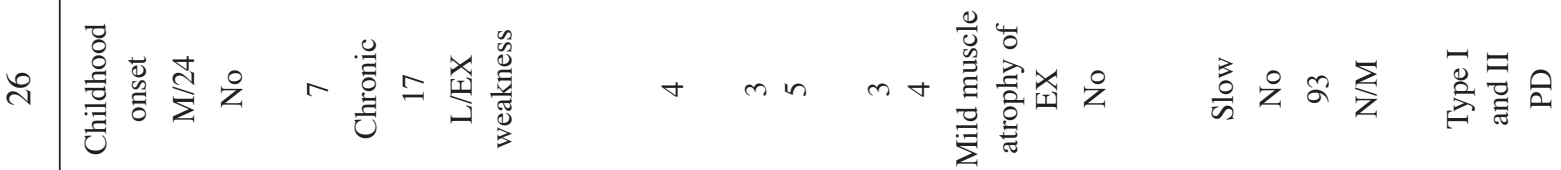

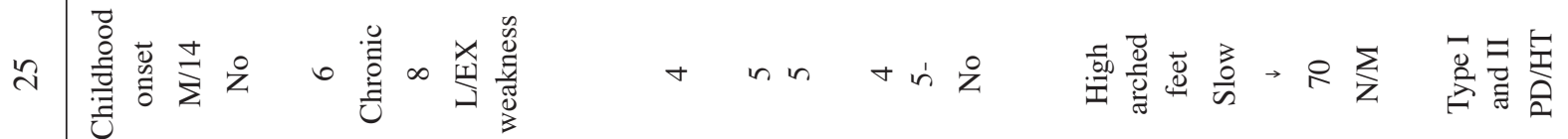

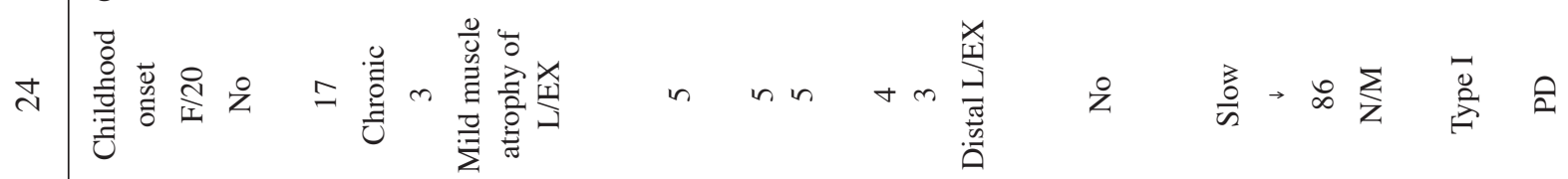

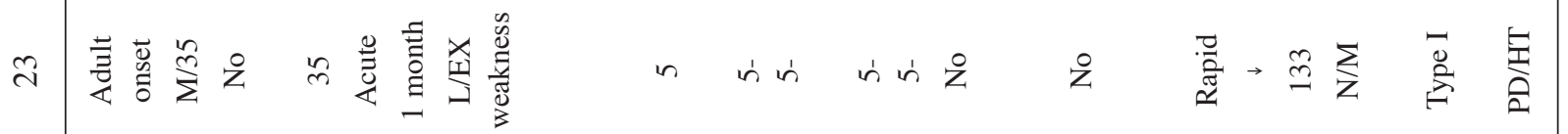

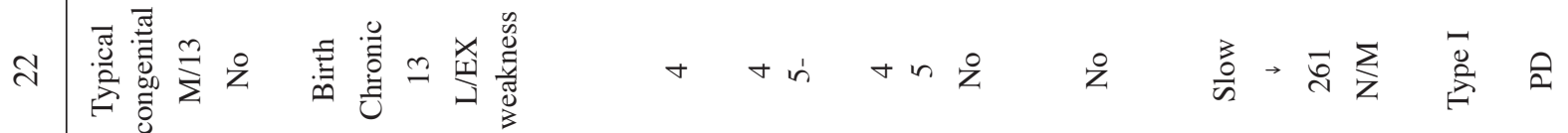

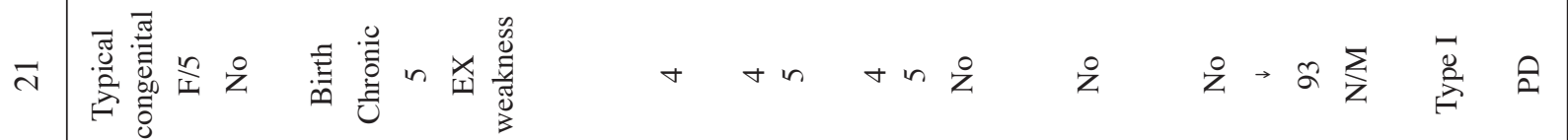

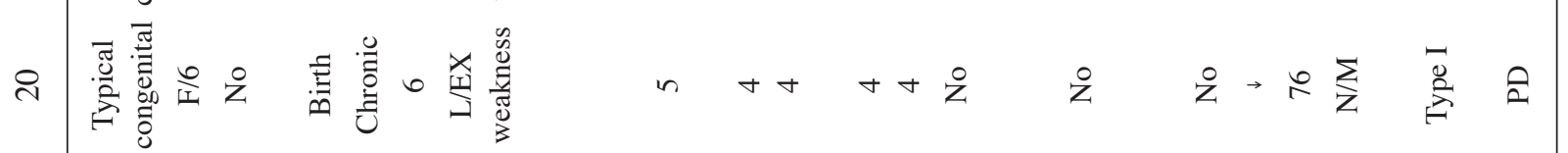

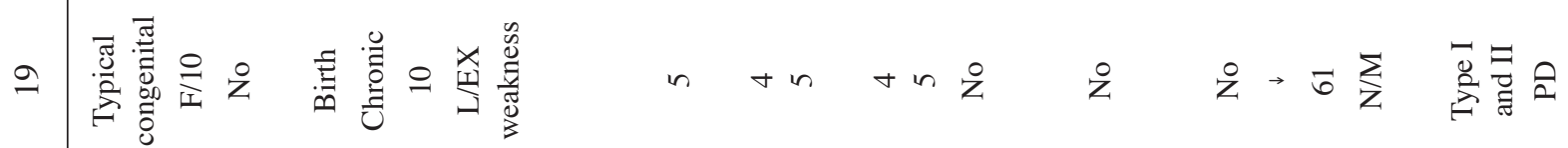

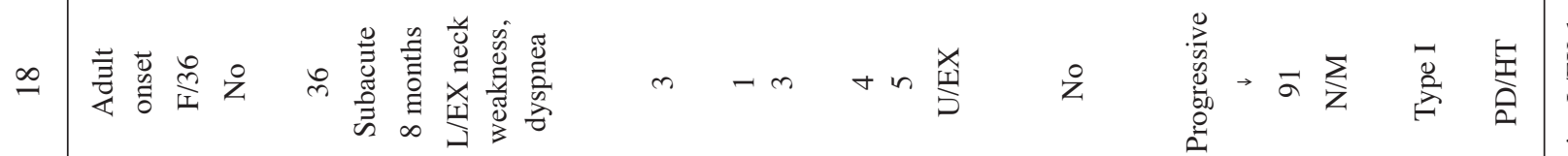

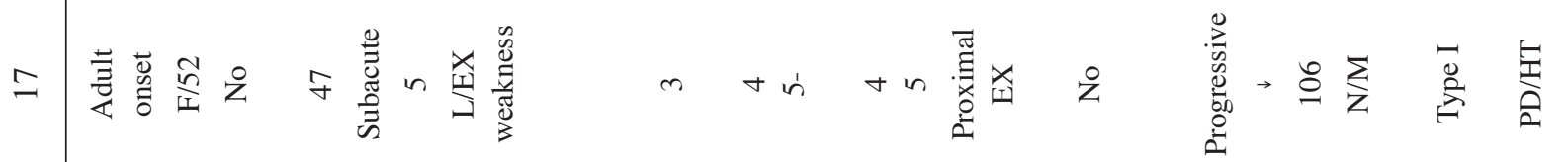

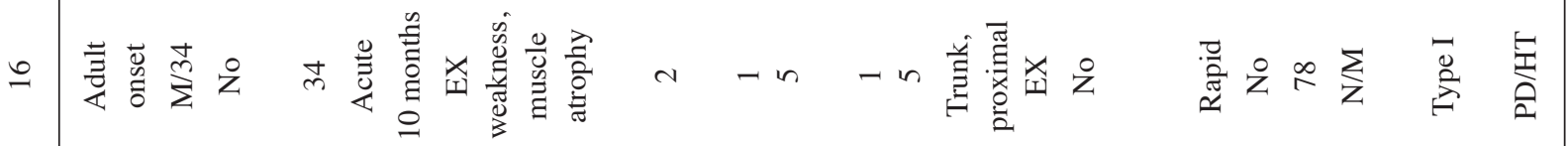
几

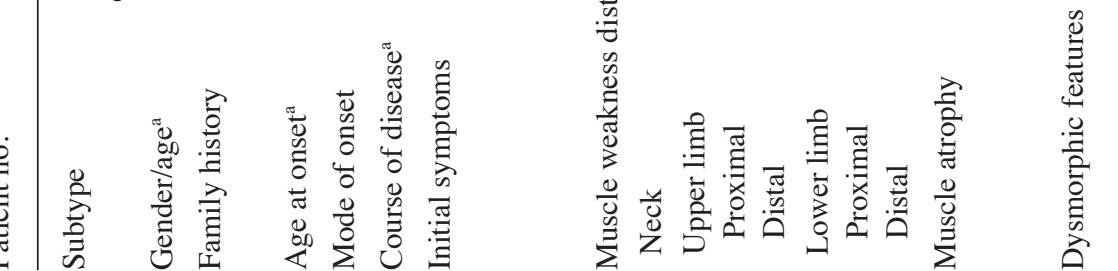

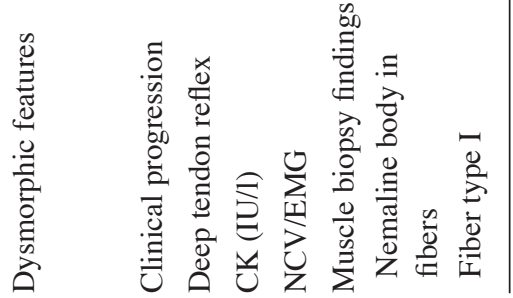

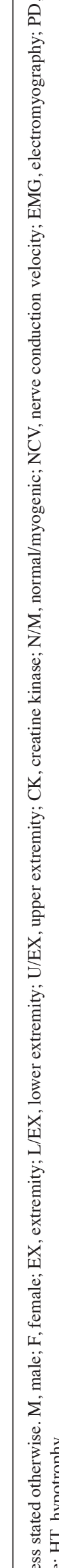




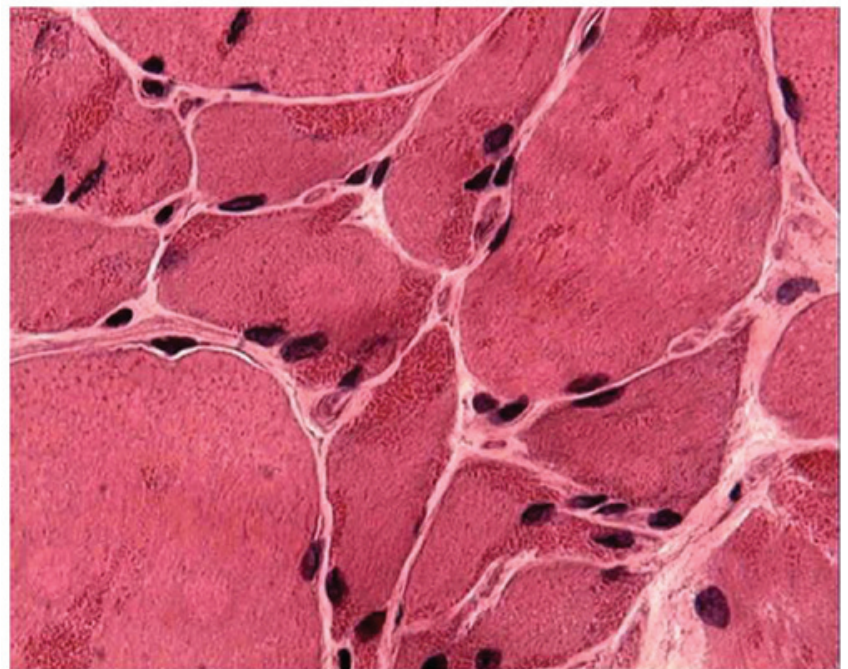

Figure 1. Haematoxylin and eosin staining (magnification, $\mathrm{x} 400$ ) shows fiber size variation, with areas of small groups of atrophic muscle fibers. A few subsarcolemmal rods are detected.

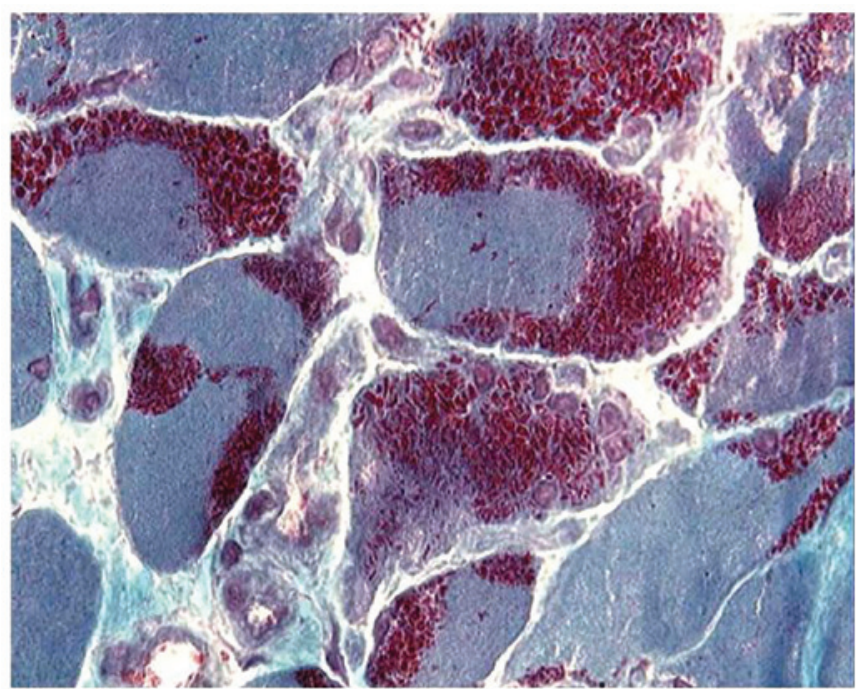

Figure 2. Modified Gömöri trichrome staining (magnification, x400) shows characteristic purple rods.

motor-unit action potentials with reduced recruitment in all patients; these are myopathic electromyography presentations. The serum CK level ranged from 28 to $261 \mathrm{IU} / 1$ (normal range 2-200 IU/l) with a mean value $111.7 \pm 60.1 \mathrm{IU} / 1$. The CK level was within the normal range for $89.3 \%$ of the patients (25/28), while it was mildly to moderately elevated in $10.7 \%$ of the patients (3/28).

Pathological information. Histological analysis of the muscles biopsies was performed in all cases, and similar abnormalities were found among the samples. Hematoxylin and eosin staining revealed variations in the fiber size, with areas of small groups of atrophic muscle fibers. A frequency of red-stained rods $>90 \%$ was found in the cytoplasm and the subsarcolemmal region of muscle fibers in 19 patients (67.9\%) (Fig. 1), while a frequency of $70 \%$ was found in $7(25 \%)$ and of $50 \%$ in $2(7.1 \%)$ patients. Some of the rods were detectable with

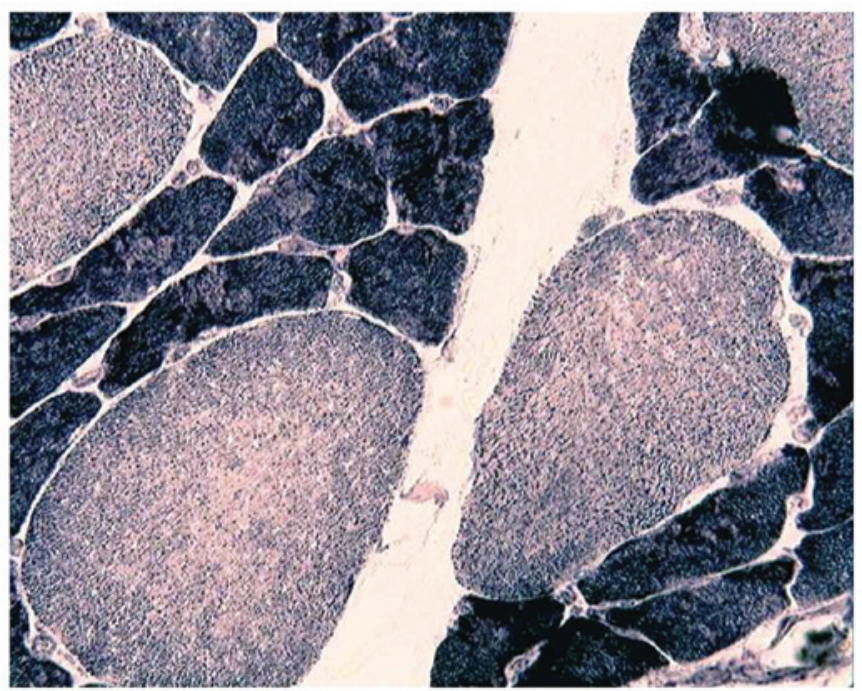

Figure 3. Nicotinamide dehydrogenase tetrazolium reductase staining (magnification, x400) shows type I fiber atrophy and core-like areas devoid of enzyme activity in some larger fibers.

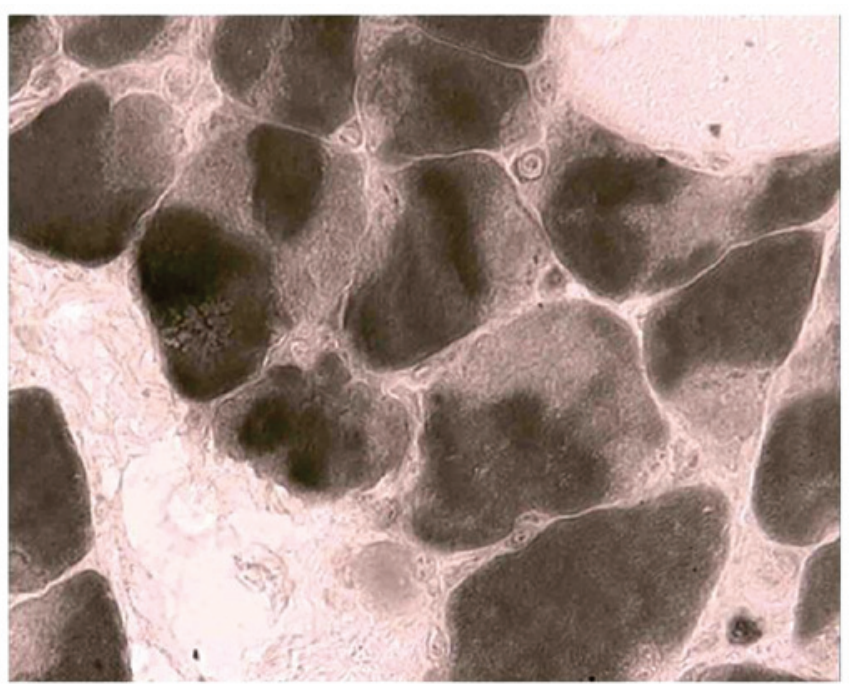

Figure 4. Adenosine triphosphatase staining at $\mathrm{pH} 4.5$ (magnification, $\mathrm{x} 400$ ) shows type I fiber predominance. Rods are confined in type I fibers.

hematoxylin and eosin, but most of them became evident upon modified Gömöri trichrome staining (Fig. 2). Characteristic purple-colored rods were observed in the cytoplasmic and subsarcolemmal region of muscle fibers stained with modified Gömöri trichrome, which were more prominent in type I fibers. ATPase stain showed the predominance of type I fibers in all patients (Fig. 3) and the type I fiber atrophy in 12 patients. Nemaline rods were confined in type I fibers in 18 patients, while rods were never observed to be confined in type II fibers. A histochemical reaction for NADH-TR showed that the darker, type I fibers are smaller in diameter than the pale, type II fibers, and revealed the presence of core-like areas devoid of enzyme activity in some larger fibers (Fig. 4). Electron microscopy allowed detection of nemaline rods as high electron-dense rod-shaped or ovoid structures; these structures located in the middle of the muscle fibers or were accompanied by a disorganized myofibrillar apparatus, with 


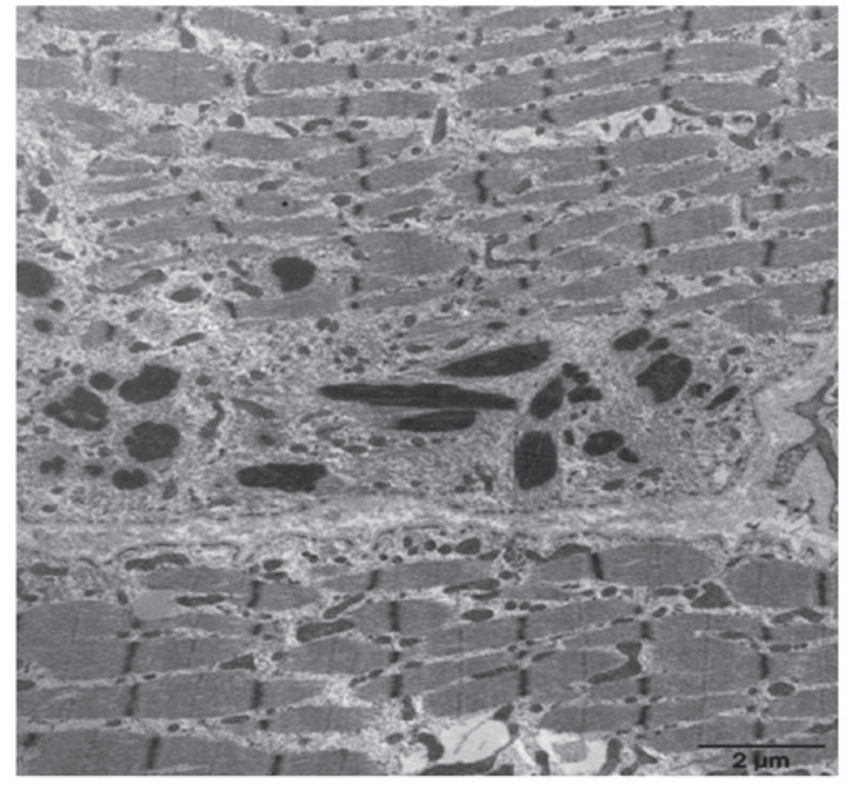

Figure 5. Electron microscopy (magnification, x10,000) shows high electron-dense rod-shaped structures, accompanied by a disorganized myofibrillar apparatus, and areas without rods with normal sarcomere appearance.

broken myofilaments, and irregular myofibrils and $\mathrm{Z}$ lines (Fig. 5).

\section{Discussion}

$\mathrm{NM}$ is a clinically heterogeneous congenital myopathy, characterized by the presence of rod-like structures in the cytoplasm of muscle fibers. The rods, or nemaline bodies, are stained purple with the modified Gömöri trichrome staining method or can be visualized by electron microscopy as high electron-dense bodies similar to Z bands. Similar to other congenital myopathies, NM was named after the Greek word 'nema' for the thread observed in muscle biopsies. Although a relatively rare disease, NM is the most common non-dystrophic congenital myopathy, with an estimated incidence of 0.02 per 1,000 live births worldwide. In China, only a few NM cases were reported, and related epidemiological data have not yet become available. In our study, we screened, using muscle biopsy, a total of 4,127 patients all over China from 1986 to 2011, and identified 12 patients with histopathological signs of NM. The proportion of NM in this pool of suspected myopathy cases is $\sim 0.29 \%$.

The clinical features of NM patients are highly variable, ranging from neonatal death to mild limb weakness. The onset age also ranges from neonates to several decade-old individuals. In 2000, according to the age of patients at onset, severity of muscle weakness, development of clinical progression and respiratory involvement, the ENMC International Consortium classified NM into six types (4). The severe congenital type is the most severe type of NM. The first symptoms manifest at birth, with hypotonia and profound muscle weakness, particularly affecting the diaphragm and intercostal muscles, and eventually leading to severe respiratory insufficiency. Patients can perform few spontaneous movements and have difficulties in swallowing and sucking. Most cases die within one year. Patients of the intermediate congenital type can perform spontaneous movements or respiration at birth, but as the disease develops, most cases become unable to achieve ambulation or respiratory independence at early childhood. Characteristic of patients of this type is that walking is performed with the help of a wheelchair before the age of 11 years. Most NM cases belong to the typical congenital type. Although muscle weakness and hypotonia occur during the neonatal period, these patients often improve strength with age and lead an independent life. The clinical features of patients of the mild childhood type are similar to those of patients of the typical congenital type, except for the onset age. The NM adult onset type comprises a heterogeneous group of patients, with the disease onset reported between the third and the sixth decade of life. Respiratory muscle involvement along the rapidly progressive disease course is characteristic of this type, and often causes respiratory dysfunction, while its is also associated with poor prognosis. The other unusual types of NM include rare features such as cardiomyopathy, ophthalmoplegia and the detection of intranuclear nemaline bodies.

Three groups of patients were included in our study: typical congenital, adult onset and mild childhood, accounting for $53.6,25$ and $21.4 \%$ of the studied population, respectively, This distribution is similar to that reported by Ryan et al (17). Our study highlighted the clinical heterogeneity of NM. On the one hand, patients of the same type shared a number of clinical features: limb weakness and delayed motor milestone at birth in the typical congenital, proximal limb weakness at early childhood in the mild childhood and rapidly progressive disease course in the adult onset type. On the other hand, there were also slight differences among patients of the same type regarding aspects such as initial symptoms, muscle weakness distribution and clinical progression. One finding from the present study that is different from previous studies conducted on patients from other countries is the high proportion of adult onset type patients (17). The reason for missing severe and intermediate type patients may relate to the small number of patients enrolled in our study, and to a bias for exclusion of neonatal and infantile patients: Patients in neurological departments are often adolescents and adults, since NM children can seldom survive long. A future study will include a higher number of NM patients to confirm the validity of our findings.

Because of the great heterogeneity of NM patients, muscle biopsy analysis used for diagnosis of NM was mainly based on the presence of rod-like structures or nemaline bodies in the muscle fibers. Haematoxylin and eosin-stained sections of skeletal muscle from patients with NM appeared normal, or exhibited a certain variation in fiber size, but staining of frozen sections with the modified Gömöri trichrome dye revealed the presence of rods, the hallmark of this disorder, which were stained purple in contrast to the pale blue-green myofibrils. Our study also showed a tendency for rods to be confined in type I fibers, in clusters under the sarcolemmal membrane, or rarely in the nuclei, which is often associated with poor prognosis (18). Similar to a previous report (19), muscle type disproportion was common, and fiber type I predominance and muscle atrophy were frequently observed in NM patients. The distribution and number of rods was variable between different muscles or different patients, and sometimes a second biopsy was needed. There was no obvious correlation between the number of rods and the clinical severity of the 
disease. A correlation between the severity of clinical features and alterations in the size and proportion of type I myofibers was previously reported (20).

When observed under an electron microscope, rods appeared as high electron-dense ovoid structures that were often parallel to the long axis of the sarcomere or located near the $\mathrm{Z}$ disc, measuring 1-7 $\mu \mathrm{m}$ in length and $0.3-2 \mu \mathrm{m}$ in width. The rods altered the normal structure of the sarcomere, while in the area without rods, the sarcomere was well organized. Although the exact source of rods is still unclear, these structures may derive from the $\mathrm{Z}$ disc. Luther and Squire (21) demonstrated with electron microscopy experiments that rods have a lattice structure similar to that of $\mathrm{Z}$ disks, while the major constituent of rods is $\alpha$-actinin, which is also the component of the $\mathrm{Z}$ disc.

Genetic studies have identified mutations in seven distinct genes that are associated with the onset of NM: slow-muscle $\alpha$-tropomyosin (TPM3) (22), nebulin (NEB) (23), $\alpha$-actin (ACTA1) (24), $\beta$-tropomyosin (TPM2) (25), troponin T1 (TNNT1) (26), cofilin-2 (CFL2) and kelch repeat and BTB domain-containing 13 (KBTBD13) (27). Except for $K B T B D 13$, the other six genes all encode protein components of the muscle thin filaments (28). Importantly, this molecular variability in NM-causing mutations extends to inheritance patterns, as mutations in at least a few of these genes have either autosomal dominant or autosomal recessive patterns of inheritance. The gene $N E B$ was localized on the human chromosome 2q21.2-q22 based on a linkage study by Wallgren-Pettersson et al (23); the gene encodes the protein nebulin, which is the main component of the axis of the thin filament. Mutations in $N E B$ are only seen in autosomal recessive cases (29) and are associated with 50\% of NM cases related to gene mutation. Moreover, most clinical cases related to $N E B$ mutations are of typical congenital type (30). Besides mutations in $N E B$, the second most common mutation associated with $\mathrm{NM}$ patients was discovered in the ACTAl gene, accounting for 10-20\% of NM cases caused by mutations (31). About half of the cases related to mutations in ACTAl are associated with the severe congenital type and display autosomal recessive, as well as autosomal dominant inheritance patterns (32). A mutation in TNNT1 was identified in Old Order Amish families with NM history, inherited in an autosomal recessive pattern (26). A mutation in the CFL2 gene, locating on $14 \mathrm{q} 12$, was reported in four cases displaying facial weakness and foot drop $(33,34)$. TPM3 mutations have been discovered in rare dominant and recessive cases of NM, and rarely are TPM2 in autosomal dominant mode (25). It is notable that nemaline bodies are only present in type I fibers of patients with TPM3 mutations, in accordance with the fact that TPM3 encodes the slow-muscle $\alpha$-tropomyosin in type I fibers. Recently, mutations in KBTBD13 have been reported to cause dominantly inherited NM (26). In contrast to the other 5 genes mutations in which cause NM, KBTB13 encodes a protein with a kelch repeat and a BTB domain, which is not related to the thin filament of the myofiber; the function of this protein is still unclear.

In conclusion, NM is a rare myopathy in China, with only $0.29 \%$ of cases detected among the screened myopathy cases in this study. The 28 Chinese patients with NM examined herein showed great heterogeneity in clinical features, and their types ranged from typical congenital and mildly affected at childhood to adult onset type with respiratory failure. The diagnosis of NM was mainly based on the presence of characteristic rods in $>50 \%$ of the muscle fibers. Genetic analyses are necessary to further investigate the pathophysiology of the observed clinical and pathological features of these patients.

\section{Acknowledgements}

This study was supported by a grant from the Characteristic Clinical Application Program of the Beijing Municipal Science and Technology Commission (no. Z111107058811108).

\section{References}

1. Agrawal PB, Strickland CD, Midgett C, et al: Heterogeneity of nemaline myopathy cases with skeletal muscle alpha-actin gene mutations. Ann Neurol 56: 86-96, 2004.

2. Conen PE, Murphy EG and Donohue WL: Light and electron microscopic studies of 'myogranules' in a child with hypotonia and muscle weakness. Can Med Assoc J 89: 983-986, 1963.

3. Shy GM, Engel WK, Somers JE and Wanko T: Nemaline myopathy. A new congenital myopathy. Brain 86: 793-810, 1963.

4. Wallgren-Pettersson C and Laing NG: Report of the 70th ENMC International Workshop: nemaline myopathy, 11-13 June 1999 , Naarden, The Netherlands. Neuromuscul Disord 10: 299-306, 2000.

5. Wallgren-Pettersson C, Sewry CA, Nowak KJ and Laing NG: Nemaline myopathies. Semin Pediatr Neurol 18: 230-238, 2011.

6. Cao P, Lu D and Feng LF: A case report of nemaline myopathy. Hered Dis 7: 243-244, 1990.

7. Li YX, Wu LJ, Chen QT, et al: Adult nemaline myopathy. J Clin Neurol 11: 369-370, 1998 .

8. Li YX, Wu LJ and Chen QT: Clinical and pathological discussion: the 42nd cases. Chin J Neurol 34: 122-124, 2001.

9. Han Y,Zheng HM, Zhou S, Deng BQ, Wu T, et al: A discussion of clinical and pathological features and pathogenesis of nemaline myopathy (NM). J Apoplexy Nerv Dis 19: 90-92, 2002.

10. Yan CZ, Liu SP, Wu JL, et al: Adult form nemaline myopathy: two cases report with clinicopathological and ultrastructural study. Chin J Neurol 36: 173-175, 2002 (In Chinese).

11. Lai HW, Yang CH, Wang FH and Tang GY: Electron microscopic observation of nemaline myopathy (report of a case). J Chin Electr Microsc Soc 23: 77-80, 2004.

12. Yuan JH, Hu J, Chen L, Mei L, Kang ZJ, et al: The clinical and pathological analysis of the nemaline myopathy complicated with the uniform type I fiber myopathy. J Apoplexy Nerv Dis 22: 432-433, 2005.

13. Chen $\mathrm{L}$ and $\mathrm{Hu} \mathrm{J}$ : A case report of nemaline myopathy. Chin $\mathrm{J}$ Pract Pediatr 21: 154, 2006.

14. Lu Y, Da Y, Wang M, Liu L and Jia JP: Nemaline myopathy - A 2 cases report and review of literatures. Chin J Neuroimmunol Neurol 15: 236-238, 2008.

15. Lu HD, Li ZF, Qin DX, Zhang SJ, Qian Q, et al: Clinical and pathological analysis of 3 nemaline myopathy cases. Chin J Neurol 41: 465-467, 2008

16. Jiang H1, Xiao B, Jia DD, Zhang N, Xu XP, et al: Clinical and pathologic analysis of an autosomal recessive kindred with nemaline myopathy. Zhonghua Yi Xue Za Zhi 89: 3316-3319, 2009 (In Chinese).

17. Ryan MM, Schnell C, Strickland CD, Shield LK, Morgan G, et al: Nemaline myopathy: a clinical study of 143 cases. Ann Neurol 50: 312-320, 2001

18. Goebel HH, Piirsoo A, Warlo I, Schofer O, Kehr S, et al: Infantile intranuclear rod myopathy. J Child Neurol 12: 22-30, 1997.

19. Gurgel-Giannetti J, Reed UC, Marie SK, Zanoteli E, Fireman MA, et al: Rod distribution and muscle fiber type modification in the progression of nemaline myopathy. J Child Neurol 18: 235-240, 2003.

20. Bhatt JR and Pascuzzi RM: Neuromuscular disorders in clinical practice: case studies. Neurol Clin 24: 233-265, 2006.

21. Luther PK and Squire JM: Muscle Z-band ultrastructure: Titin Z-repeats and Z-band periodicities do not match. J Mol Biol 319: 1157-1164, 2002. 
22. Laing NG, Wilton SD, Akkari PA, Dorosz S, Boundy K, et al: A mutation in the alpha tropomyosin gene TPM3 associated with autosomal dominant nemaline myopathy NEM1. Nat Genet 10: 249, 1995.

23. Wallgren-Pettersson C, Avela K, Marchand S, et al: A gene for autosomal recessive nemaline myopathy assigned to chromosome $2 \mathrm{q}$ by linkage analysis. Neuromuscul Disord 5: 441-443, 1995.

24. Nowak KJ, Wattanasirichaigoon D, Goebel HH, Wilce M, Pelin K, et al: Mutations in the skeletal muscle alpha-actin gene in patients with actin myopathy and nemaline myopathy. Nat Genet 23: 208-212, 1999.

25. Wattanasirichaigoon D, Swoboda KJ, Takada F, Tong HQ, Lip V, et al: Mutations of the slow muscle alpha-tropomyosin gene, TPM3, are a rare cause of nemaline myopathy. Neurology 59: 613-617, 2002

26. Johnston JJ, Kelley RI, Crawford TO, Morton DH, Agarwala R, et al: A novel nemaline myopathy in the Amish caused by a mutation in troponin T1. Am J Hum Genet 67: 814-821, 2000.

27. Sambuughin N, Yau KS, Olivé M, Duff RM, Bayarsaikhan M, et al: Dominant mutations in KBTBD13, a member of the $\mathrm{BTB} /$ Kelch family, cause nemaline myopathy with cores. Am J Hum Genet 87: 842-847, 2010.

28. Sanoudou D and Beggs AH: Clinical and genetic heterogeneity in nemaline myopathy - a disease of skeletal muscle thin filaments. Trends Mol Med 7: 362-368, 2001.
29. Ottenheijm CA, Hooijman P, DeChene ET, Stienen GJ, Beggs AH, et al: Altered myofilament function depresses force generation in patients with nebulin-based nemaline myopathy (NEM2). J Struct Biol 170: 334-343, 2010

30. Wallgren-Pettersson C, Pelin K, Nowak KJ, Muntoni F, Romero NB, et al: Genotype-phenotype correlations in nemaline myopathy caused by mutations in the genes for nebulin and skeletal muscle alpha-actin. Neuromuscul Disord 14: 461-470, 2004.

31. Visegrády B and Machesky LM: Myopathy-causing actin mutations promote defects in serum response factor signaling. Biochem J 427: 41-48, 2010.

32. Laing NG, Dye DE, Wallgren-Pettersson C, Richard G, Monnier N, et al: Mutations and polymorphisms of the skeletal muscle alpha-actin gene (ACTA1). Hum Mutat 30: 1267-1277, 2009.

33. Agrawal PB, Greenleaf RS, Tomczak KK, et al: Nemaline myopathy with minicores caused by mutation of the CFL 2 gene encoding the skeletal muscle actin-binding protein, cofilin-2. Am J Hum Genet 80: 162-167, 2007.

34. Ockeloen CW, Gilhuis HJ, Pfundt R, et al: Congenital myopathy caused by a novel missense mutation in the CFL2 gene. Neuromuscul Disord 22:632-639, 2012. 